\title{
Use of dynamic contrast enhanced time intensity curve shape analysis in MRI: theory and practice
}

This article was published in the following Dove Press journal:

Reports in Medical Imaging

30 July 2013

Number of times this article has been viewed

\author{
Cristina Lavini' \\ Maarten S Buiter ${ }^{2}$ \\ Mario Maas' \\ 'Department of Radiology, Academic \\ Medical Center, Amsterdam, \\ The Netherlands; ${ }^{2}$ Department \\ of Radiotherapy, The Netherlands \\ Cancer Institute, Amsterdam, \\ The Netherlands
}

\begin{abstract}
The analysis of dynamic contrast enhanced data using the classification of the time intensity curve (TIC) shape is widely employed both in its region of interest and pixel by pixel variants. While its application in breast imaging is established and documented by a large amount of scientific works, its use for other body parts is still scattered and there is no consensus as to whether the method can be used alone to perform differential diagnosis in cancer or in inflammatory diseases. In this review we evaluate all the literature which makes use of TIC shape analysis in tissues other than breast, discuss the results, highlight the possible shortcomings, and suggest directions for future research.
\end{abstract}

Keywords: DCE-MRI, TIC shape, pattern recognition, review

\section{Introduction}

Dynamic contrast enhanced magnetic resonance imaging (DCE-MRI) is an important imaging technique used in radiology as an additional tool in oncology assessment. Its safety, repeatability, high spatial resolution, and the fact that it can be performed on clinical magnetic resonance imaging (MRI) scanners with standard specifications allow its application in routine clinical and research settings. The additional value of this technique, with respect to conventional static MR imaging sequences, lies in its ability to identify changes in tissue physiology when changes in the anatomy are not yet visible. In the last two decades it has become an established method, and it is now often performed as part of a routine MRI protocol.

Technically, DCE-MRI consists of a series of fast MRI scans, commonly available on all clinical scanners, which are acquired for a duration of 3 to 10 minutes while a gadolinium (Gd)-based contrast agent is injected intravenously. As the contrast medium flows from the blood pool into the tissue, the signal intensity on a T1-weighted image during and after the injection varies according to a pattern which is dependent on the vascularization and on the viability of the tissue to the contrast agent. This time dependent signal intensity or time intensity curve (TIC) in the tissues is recorded and further analyzed to provide several parameters useful for diagnosis.

The analysis of data generated by DCE-MRI is not fully standardized yet, and can roughly be divided into three general approaches: visual assessment of the TIC curves, parametric analysis of the time dependent MRI signal, and quantitative analysis using pharmacokinetic models (PKM).
Correspondence: Cristina Lavini Department of Radiology, Academic Medical Center, Meibergdreef 9, I I00DE Amsterdam, The Netherlands

$\mathrm{Tel}+3 \mid 205666519$

Fax +31 205669119

Email c.lavini@amc.uva.nl 


\section{Visual TIC shape assessment}

A common approach in the analysis of DCE-MRI is to look at the dynamic data by selecting a region of interest (ROI) in the lesion and to observe how the average signal intensity of the ROI varies with time. Based on this observation, the radiologist can spot areas of vascular disruption, which are characterized by a quick signal uptake. This approach, although operator dependent, is widely used in routine clinical practice, especially in breast imaging. The visual assessment of the shape of the original TIC has some obvious advantages: it is easy to perform and it can be easily applied in daily clinical routine.

\section{Parametric analysis of the signal enhancement}

Parametric analysis involves the generation of parameters describing the original DCE-MRI signal intensities, such as the maximum enhancement (ME), rate of enhancement, time to peak, initial area under the curve, etc. It can be calculated on a pixel by pixel (or more strictly speaking, voxel by voxel) basis and rendered in parametric images. Several commercially available software packages allow this type of analysis. Unfortunately, the calculated parameters are dependent on the MRI protocol chosen and are not quantitative, ie, they are not a measure of intrinsic physiological properties. The parameters used in the T1-w MR sequence, eg, the repetition time (TR) and flip angle, significantly affect the relationship between the amount of contrast agent in the tissue and the signal intensity change. As a result, the same amount of Gd uptake in the tissue might result in significantly varying relative signal change in differently weighted MR sequences, as nicely exemplified by Evelhoch. ${ }^{1}$ The technique is, therefore, not suited to comparing disease activity across patients in clinical studies.

\section{PKM}

Quantification of the DCE-MRI data can be achieved by means of PKM based analysis. ${ }^{2}$ The application of the theoretical PKM to the DCE-MRI data (through parameter fitting) permits the extraction of physiologically relevant quantities that reflect intrinsic properties of the tissue, such as $\mathrm{v}_{\mathrm{e}}$ (the extracellular extravascular space), $\mathrm{K}^{\text {trans }}$ (the forward transfer coefficient of Gd between plasma and $v_{\mathrm{e}}$ ), and $\mathrm{v}_{\mathrm{i}}$ (the vascular volume). Nowadays, this is an established method which has gained trust and popularity among scientists for its ability to grade cancer, and to assess neoangiogenesis and the effect of drugs. ${ }^{3,4}$ Unlike most MRI based techniques, it is (or it strives to be) quantitative, ie, it measures intrinsic properties of the tissue. However, the very complex implementation of PKM, as well as its considerable propensity for errors, has made it very challenging to apply it in clinical practice. Examples of these challenges include the fact that the model requires the knowledge of the absolute tissue contrast agent concentration, whose calculation requires the measurement of the native T1 maps, as well as knowledge of the arterial input function (AIF), a fundamental input of the model. Moreover, the accurate sampling of the vascular signal used to generate the AIF puts some strict constraints on the minimal temporal resolution of the dynamic scan. ${ }^{5}$

In this review we will concentrate on the methods based on (or derived from) the first of these three aforementioned analysis approaches, and we refer to that as the "TIC shape analysis."

\section{TIC shape analysis}

Since the introduction of DCE-MRI in breast imaging, ${ }^{6}$ radiologists have observed and classified a number of enhancement shape types in various pathologies (Figure 1). In DCE-MRI of the breast, the dynamic scan consists of a few dynamic scans (usually three), each scan being repeated every 90 seconds. ${ }^{7}$ The difference between the various shapes is found in the different slopes of the line connecting the three points in the image (Figure 1A). In other body parts, the dynamic scans are usually acquired with a higher temporal resolution, and the shapes of the TIC can vary across a wider range of patterns (Figure 1). ${ }^{8,9}$ The TICs are usually obtained from a selected ROI, encompassing the whole or a part of the lesion.

Although not an absolute measure of permeability, the shape of the uptake curve of a dynamic scan is a reflection of the tissue viability and permeability to the contrast agent; the rise in contrast enhancement reflects (indirectly) the transfer of the contrast agent from the capillary to the extravascular space, from which the agent is later reabsorbed by the blood pool, resulting, eventually, in a signal decrease. This increasing-decreasing pattern reflecting higher capillary permeability can be observed within the typical duration of the DCE-MRI scan (5-10 minutes). Areas of lower perfusion and permeability tend to enhance in a much slower fashion, and the signal does not decrease until after the end of the DCE-MRI scan. The use of these features to make clinical decisions has led many authors to identify TIC shapes (up to seven) in the analyzed tissues and to investigate their value as a potential diagnostic feature. ${ }^{9-11}$

This straightforward approach, sometimes described as heuristic (ie, obtained by exploration of possibilities rather 

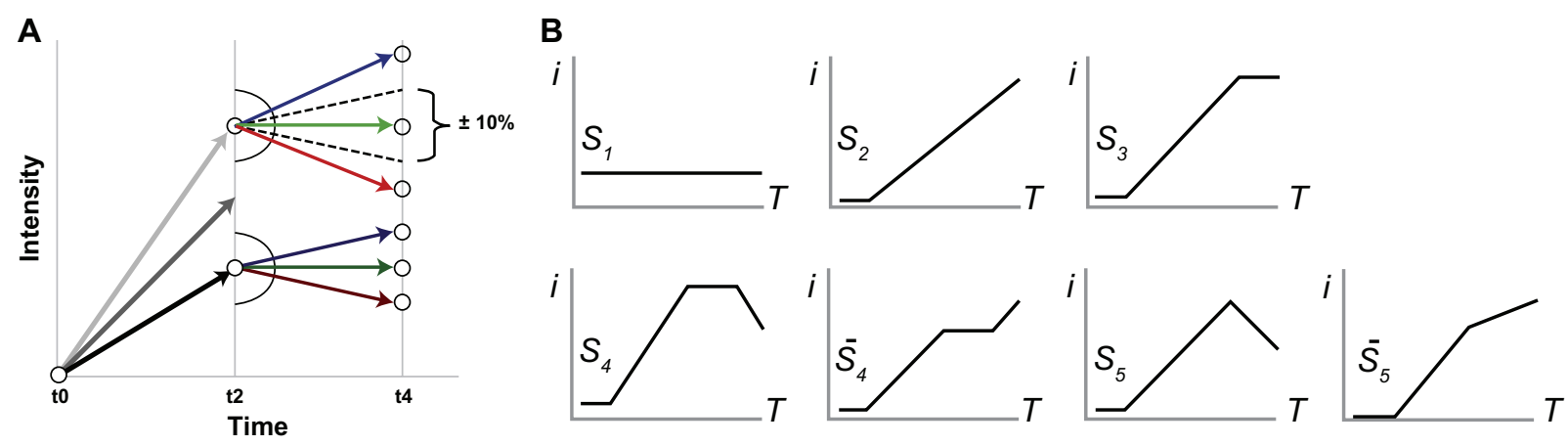

C

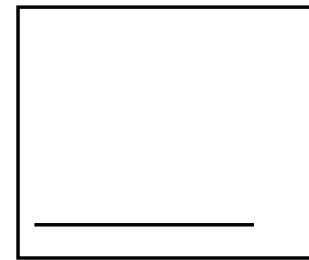

Type I

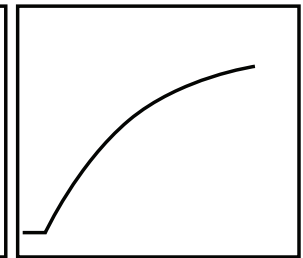

Type II

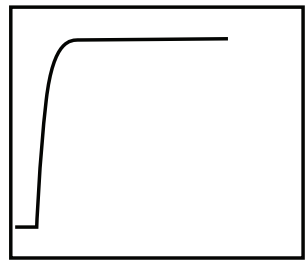

Type III

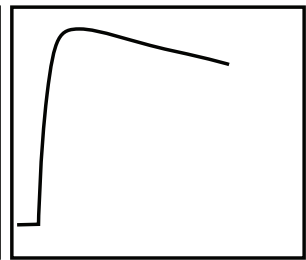

Type IV

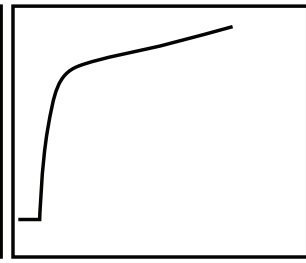

Type V

Figure I Different classification of the time intensity curve shape.

Notes: (A) Color coding scheme of the three time point method in breast. The initial rates of enhancement in the time interval between the precontrast image at time $=0$ ( $\mathrm{t} 0$ ) and the postcontrast image at time 2 (t2) are coded by color intensity (hue). (B) SI curves: $\mathrm{S}_{1}-\mathrm{S}_{5}$. (C) Time intensity curve enhancement types. (A) Reprinted from Clinical Imaging, 30(3), Hauth EA, Stockamp C, Maderwald S, et al, Evaluation of the three-time-point method for diagnosis of breast lesions in contrast-enhanced MR mammography, 160-165, Copyright 2006, with permission from Elsevier. (B) Reprinted from Academic Radiology, 14, Kubassova O, Boyle R, Radjenovic A, Quantitative analysis of dynamic contrast enhanced MRI datasets of the metacarpophalangeal joints, I I89-1200, Copyright 2007, with permission from Elsevier. (C) Reprinted from Skeletal Radiology, 30(I), van Rijswijk CS, Hogendoorn PC, Taminiau AH, et al, Synovial sarcoma: dynamic contrast-enhanced MR imaging features, 25-30, Copyright 200I, with kind permission from Springer Science and Business Media.

Abbreviations: S, shape; i, intensity; T, time; SI, signal intensity.

than by following set rules) might lack precision and not be a quantitative measure of physiological properties, yet the number of articles using the TIC shape as a potential mirror of disease seems indeed to have grown in recent years, although predominantly in breast imaging.

Furthermore, in the last 6 years, a new line of research has taken root, ie, redeveloping the heuristic shape analysis method into something more robust, less user dependent, less MRI protocol dependent, and image-wise, enough advancement to compete with other more quantitative methods.

Instead of an ROI dependent evaluation of averaged TICs, the TICs are analyzed in a pixel by pixel (or voxel by voxel) fashion, in every single voxel acquired by the DCE-MRI scan sequence..$^{8,12,13}$ This method is proposed to overcome the intrinsic insensitivity to spatial heterogeneity of the ROI based analysis. As large lesions, whether cancerous or inflammatory, are not homogeneous, sampling and averaging signals from the ROIs to look at the dynamic course of the TIC misses important characteristics of the lesion. The pixel by pixel computer assisted analysis is done using different algorithms and its results are rendered in a color coded map (Figure 2). Tissue heterogeneity is more adequately assessed using this pixel by pixel analysis due to its higher sensitivity to spatial changes in TIC shapes.
It is the purpose of this review to present the results of works using the TIC shape as an endpoint parameter for the determination of disease. Studies where the TIC is described but not used as the main decision making tool have been omitted. Studies have been selected with a similar DCE-MRI

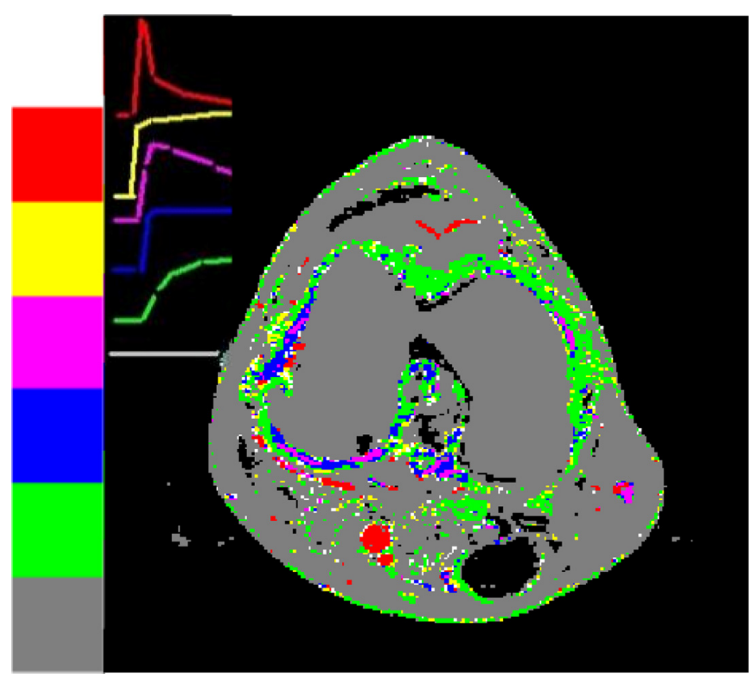

Figure 2 Pixel by pixel analysis and color rendering of the time intensity curve shape analysis. ${ }^{12}$

Note: Reprinted from Magnetic Resonance Imaging, 25, Lavini C, de Jonge MC, van de Sande MG, et al, Pixel-by-pixel analysis of DCE MRI curve patterns and an illustration of its application to the imaging of the musculoskeletal system, 604-6I2. Copyright 2007, with permission from Elsevier. 
protocol in terms of scan duration and acquisition frequency. Because the shape does depend on the time window used (all the TICs will present with a washin and washout phase if scanning time is long enough), we limited the analysis to protocols with a minimum acquisition time of 2.5 minutes, and a maximum interval between the dynamic scans of 30 seconds.

Furthermore, although the literature about TIC shape in breast MRI is very extensive (breast was the first application of TIC shape analysis in DCE-MRI ${ }^{6}$ ) and outnumbers by far the literature of TIC shape analysis in other pathologies, the largest part of the literature refers to dynamic data acquired with a low temporal sampling rate (the three point measurement ${ }^{7,14-17}$ where only three time points are acquired to favor spatial resolution). For this reason, in this review they are excluded from the analysis. A good overview of DCE-MRI methods in the breast can be found in Turnbull. ${ }^{18}$

\section{Review of clinical applications}

An overview of the articles making use of TIC shape analysis and their results is shown in Table 1 . The articles reviewed were first selected using broad search term queries in PubMed (search term [all fields]: DCE-MRI). Exclusion criteria were: breast DCE-MRI, use of PKM, use of quantitative or semiquantitative analysis methods, and time resolution of the DCE-MRI scan > 30 seconds. Articles were further selected by manually searching if the authors presented a TIC shape type focused analysis.

Applications of the technique encompass a large range of diseases and anatomy, from tumors to arthritis, from brain to musculoskeletal, rectum, liver, brain, parotid glands, etc.

A problem when comparing literature describing TIC shapes is that almost all studies use their own classification and naming of TIC shapes (see Figure 1B and C for examples).

As the number of classes (varying from 2 to 7 ) and classification names are not consistent across publications (see Figure 1), to simplify reading, we will use in this review the following two letter nomenclature, in which the first and second letter represents, respectively, the initial and the final behavior of the DCE-MRI uptake curve, and where upper and lower case letter represent, respectively, a growing and a decreasing pattern $(\mathrm{S}$ or $\mathrm{s}=$ slow, $\mathrm{F}$ or $\mathrm{f}=$ fast, $\mathrm{P}=$ plateau, $\mathrm{O}=$ absence of uptake) (Figure 3).

In some articles, type Ff is (sub) divided into two groups, depending on the velocity of the upslope. In this review we will call them Ff1 and Ff2 (Ff1 = slower washin and Ff2 = faster washin).
Regarding the choice of classes, from the overview in Table 1, it can be seen that TIC types SS and Ff are used in most studies (26 and 28 of 29 studies, respectively), followed by FP which is found in 21 of 29 studies. In 19 of 29 studies, type $\mathrm{O}$ (nonenhancing) is used, and 15 of 29 studies use type FS. Type X (enhancing but with unidentified shape) is only used by Lavini et al. ${ }^{12,23}$ Type $\mathrm{V}$ is identified by Lavini et al ${ }^{12,23}$ and Eida et al. ${ }^{26}$ However, Eida et $\mathrm{a}^{26}$ describes the shape, but does not attribute it to a vascular signal. In Figure 2, this shape as identified by Eida et al, ${ }^{26}$ has been described as Ff2. Also, Yabuuchi et $\mathrm{al}^{27}$ and Sasaki et $\mathrm{al}^{24}$ differentiate between fast and slow washout type Ff TICs (Ff1 and Ff2). Eida et al, ${ }^{26}$ classified as type $\mathrm{O}$ (as in their TIC shape illustration) everything which is not classified as SS, Ff, and V. The overview shows, on the one hand, the wide application of this technique in terms of pathologies, but also at the same time, the plethora of different classification types and conclusions.

\section{TIC analysis methods: $\mathrm{ROI}$ versus pixel by pixel}

Early works using TIC shape analysis were ROI based: a ROI was drawn by the radiologist on a suspected area, and the TIC derived from an averaged signal. Despite the general expectation that most malignant lesions tended to present a fast enhancing TIC (whereas benign lesions enhance slowly), a large overlap on the shape types across lesions was observed, ${ }^{19}$ suggesting a poor positive and negative predictive value of the TICs.

This lack of reproducibility could be attributed to various reasons. The fact that the analysis is based on an ROI, arbitrarily chosen by a radiologist on the basis of a native T1-w or postcontrast T1-w image, could result not only in subjectivity in the ROI choice, but also in signal averaging over the ROI. The information on the heterogeneity of the lesion in terms of TIC shapes was, therefore, lost.

To overcome these limitations, a new approach was proposed independently by Kubassova et $\mathrm{al}^{8}$ and by Lavini et $\mathrm{al}^{12}$ where the TICs were analyzed on a pixel by pixel basis. Other similar approaches have been proposed by others, such as Eida et $\mathrm{al}^{26}$ and Yuan et $\mathrm{al}^{13}$, who added the ME information by means of a color hue in a fashion similar to that used for breast imaging by Preim et $\mathrm{al}^{17}$ and by Sasaki et al. ${ }^{24}$ In these works, every single pixel is assigned to a specific TIC shape (according to some predefined classes) by means of a computer aided pattern recognition (PR) method and the result rendered in color coded maps. The classification is usually done by using thresholds on the various features identified to describe the TIC shape, such as the initial slope, 


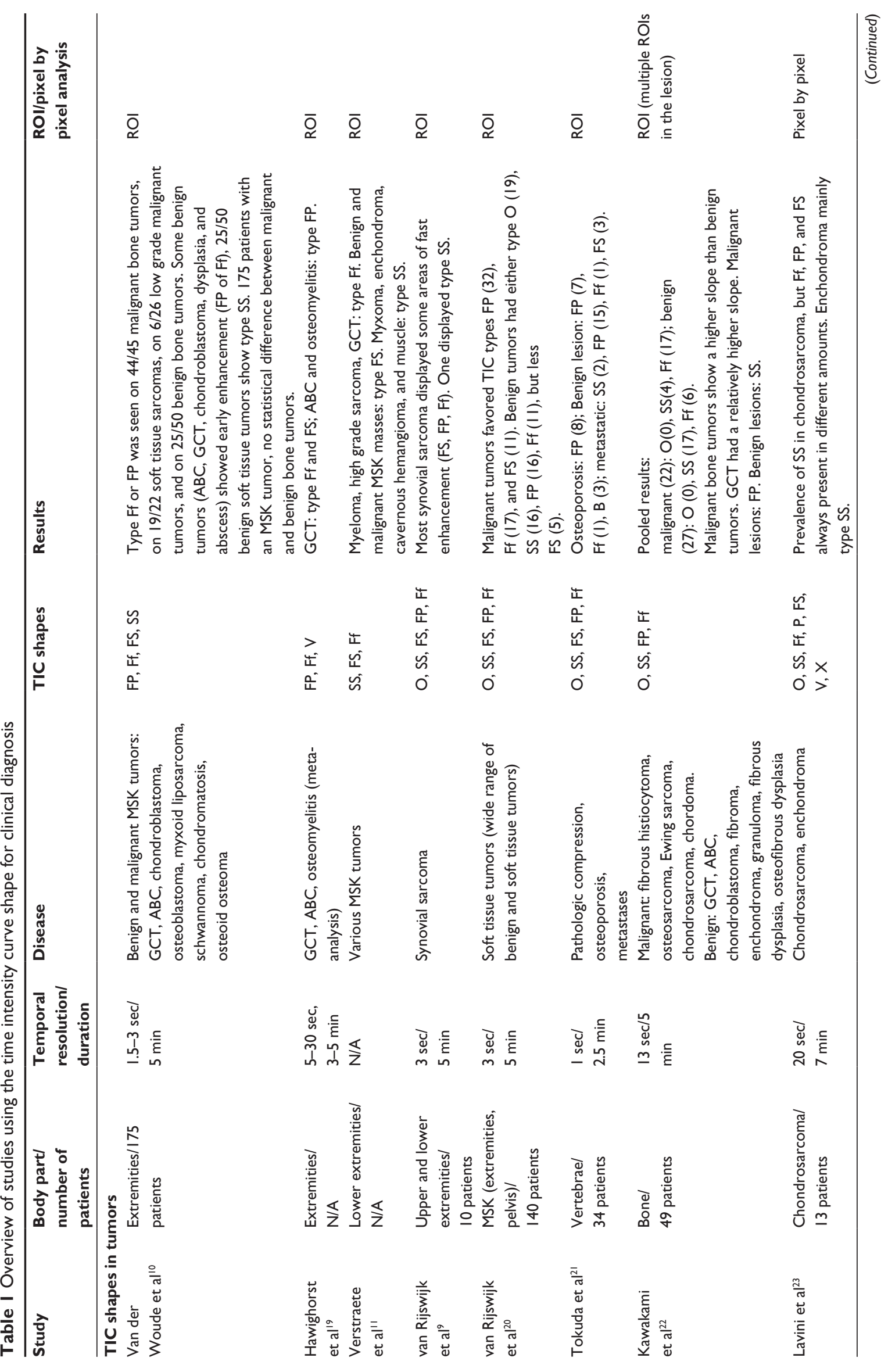




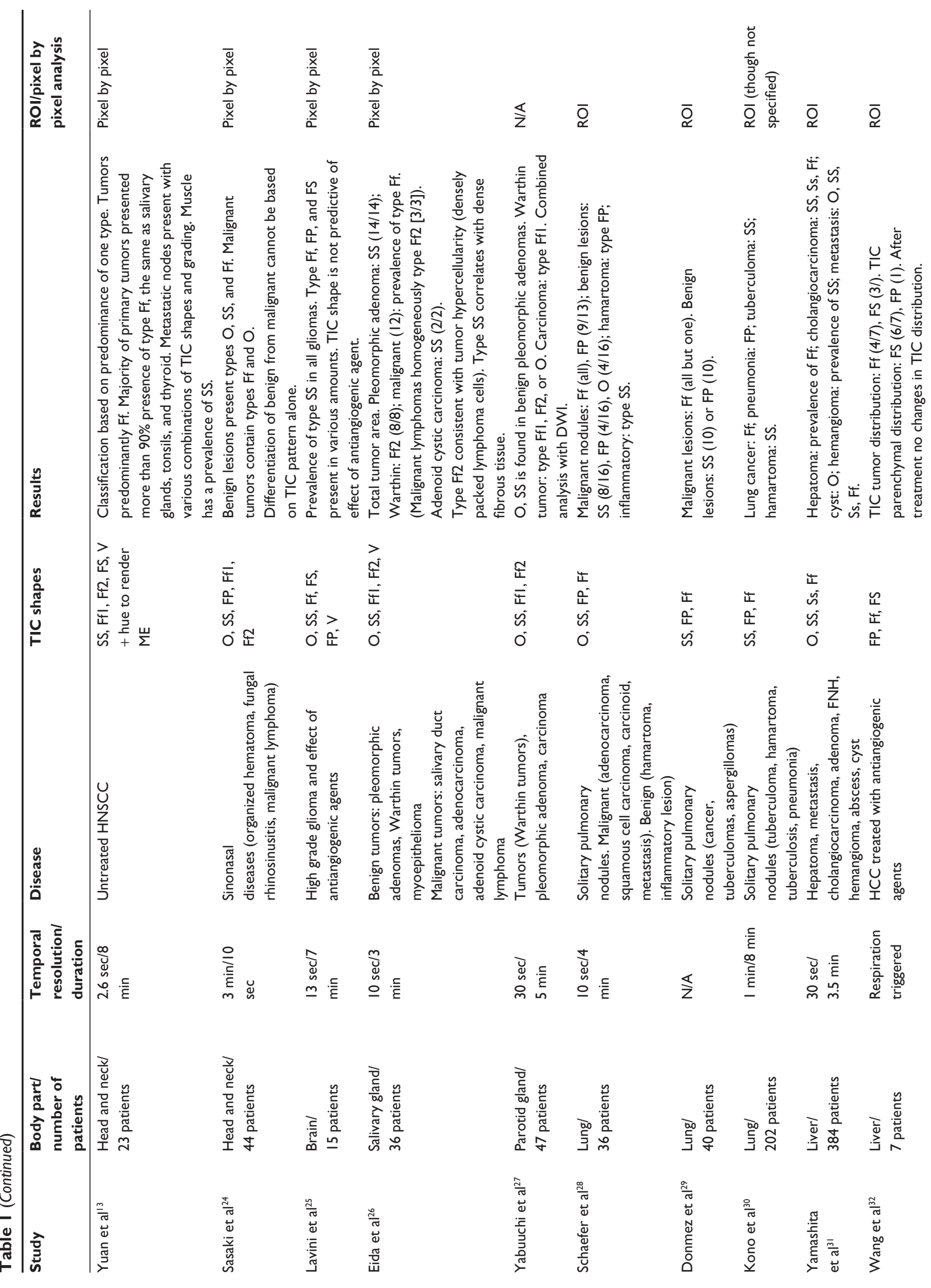




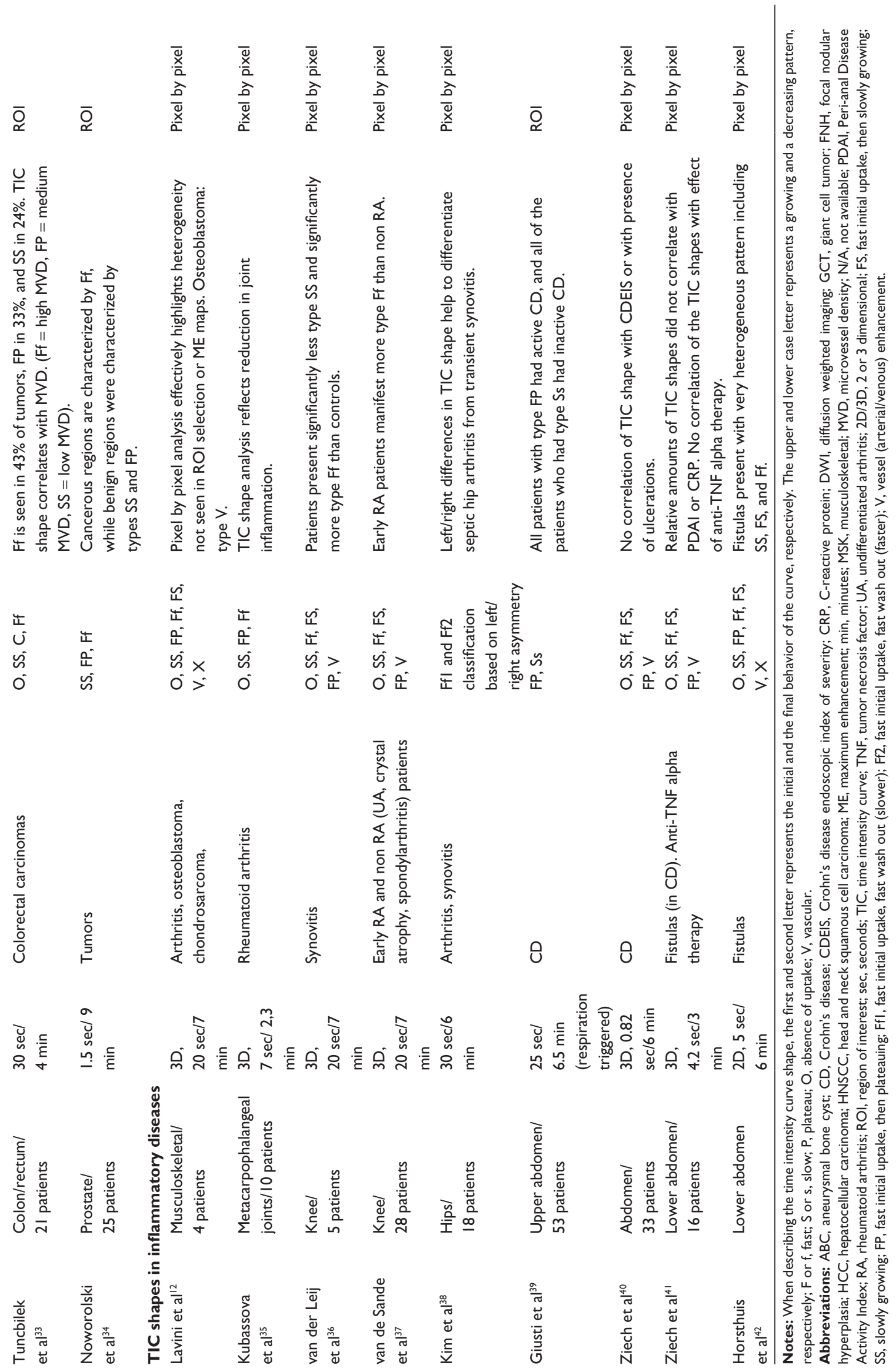




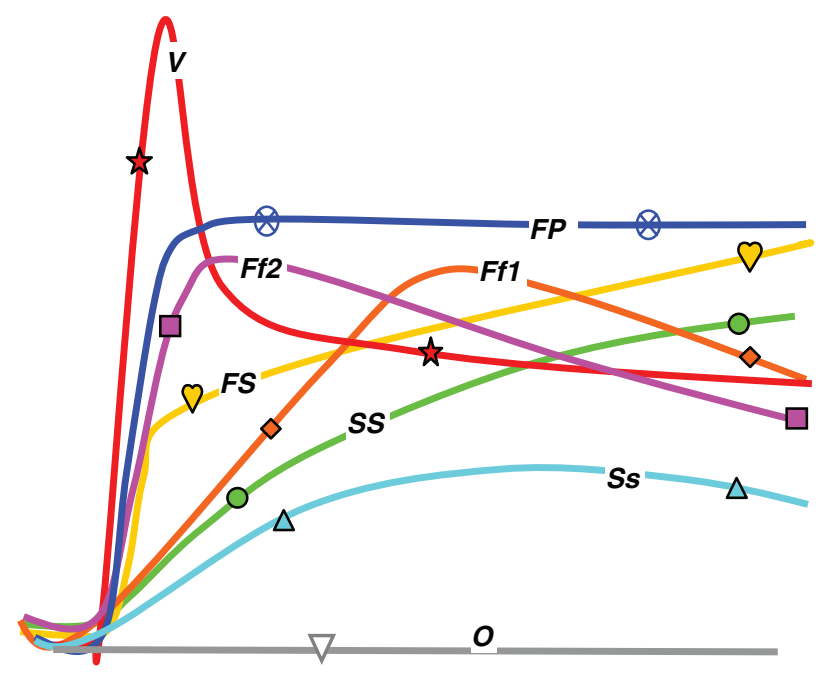

Figure 3 Proposed classification naming.

Notes: $\nabla$ Type $\mathbf{O}=$ no uptake; $\bigcirc$ Type SS: slowly growing; $\triangle$ Type Ss: slowly growing, late wash out phase; Type FS: fast initial uptake, then slowly growing; $\otimes$ Type FP: fast initial uptake, then plateauing; Type FfI fast initial uptake, fast wash out (slower); - Type Ff2 fast initial uptake, fast wash out (faster); * Type V: Vessel (arterial/venous) Enhancement; Type $\boldsymbol{X}=$ other (not shown).

the peak time, and the washout slope. ${ }^{12,24}$ Less user dependent methods such as principal component analysis (PCA) have also been used, although these methods do not rely on the use of some predefined classes (or TIC shapes) but identify the classes directly from the data. ${ }^{43}$ Because these classes cannot easily be compared with the predefined classes as described in the articles in Table 1, articles making use of component analysis were therefore not included in this review.

In the studies where a pixel by pixel approach was used, it became apparent that lesions are typically characterized by more than one TIC type, ${ }^{23}$ something that the ROI approach was not able to highlight.

Besides adding the spatial information and therefore also the visual rendering of the tissue heterogeneity in terms of dynamic behavior, the results of the pixel-by-pixel analysis lend well to the analysis of the statistical distribution of the classes. In Lavini et $\mathrm{al}^{23}$ and van der Leij et $\mathrm{al}^{36}$ the ratio between the different types of shapes is used as a possible clinical diagnostic tool. Yuan et $\mathrm{al}^{13}$ propose a classification method for which tissues are classified on the basis of the predominance of a certain shape. Tissues were then categorized as belonging to any of eight "grades" (0 to 8 ) according to the predominance of a certain shape, or, conversely, on the tendency to have evenly distributed class types.

\section{Clinical findings}

The studies presented in the review (Table 1) vary greatly in set up, purpose, and size of the cohort. Some articles presented extensive patient cohorts ${ }^{4,5,18,23,25}$ and others concentrated on one single pathology.

The majority of the studies presented in Table 1 applied the TIC shape analysis to try and differentiate between different tumors and tumor grading, others used it to investigate the activity of inflammatory processes, ${ }^{36,40}$ and others to assess the effect of drugs. ${ }^{25,32,41}$ The majority of the studies were done in the musculoskeletal (MSK) system investigating bone tumors, soft tissue tumors, and inflammatory processes (rheumatoid arthritis, synovitis). Other studies included one brain study (glioma), ${ }^{23}$ one study in prostate, ${ }^{34}$ two in liver,,${ }^{31,32}$ five in the abdomen, ${ }^{27,39-42}$ three in lungs, ${ }^{28-30}$ two in parotid/salivary glands, ${ }^{26,27}$ and two in the head and neck region. ${ }^{13,24}$

The conclusions presented are spread across a range of findings: some find the technique valuable in differentiating disease and some draw negative conclusions. Because of the large range of pathologies studied, it is not possible to pool the results. Moreover, each tumor/inflammatory process works differently and it is not easy to generalize one type of behavior in one tissue to the behavior in another tissue. Nevertheless, in the overview in Table 1, it can be seen that all 18 studies investigating cancerous lesions associated a malignant lesion with type Ff, and six of 18 associated it with type FP., $90-23,25$ Five of 18 studies s, $^{9,23,25,32}$ reported the presence of type FS in malignant lesions, and four of 18 studies reported the presence of type SS, 9,23,25,31 although these were either not predominant, or only seen in a minority of patients. Type $\mathrm{O}$ was found to occur in malignancy only in two studies. ${ }^{22,24}$

Of the 14 studies investigating benign tumors, twelwe described either the presence or a prevalence of type SS. The studies that did not associate benign tumors with type SS were describing giant cell tumors (GCT), which were associated with type Ff and FS in three studies. ${ }^{10,11,22}$ Other benign lesions (besides GCT) were also associated with type Ff and/or FP in, respectively, five $e^{10,11,20,22}$ and $\operatorname{six}^{10,12,21,28,29,34}$ of eleven cases. Interestingly, both studies investigating the (benign) Warthin tumors ${ }^{26,27}$ reported a type Ff2, making them indistinguishable from malignant lymphomas. Type $\mathrm{O}$ was found to occur in benign tumors in four studies. ${ }^{20,22,27,31}$

Metastasis was investigated in two studies, ${ }^{13,21}$ both reporting the presence of more TIC shapes (FP, Ff, FS) in various metastatic nodes. In one study, ${ }^{33}$ the correlation between the shape type and microvessel density (MVD) was presented, with the results showing that type Ff correlates with high MVD.

In one article, ${ }^{21}$ a patient with osteoporosis was presented and the resulting TIC shape assigned to type FP. Nine papers 
report findings in inflammatory processes: arthritis, ${ }^{12,35-38}$ Crohn's disease, ${ }^{39,40}$ and fistulas. ${ }^{41,42}$

As shown in Table 1, while TIC shape analysis has been applied in a wide range of diseases, it seems to have found more impact in various MSK diseases, where it was widely tested in tumors and arthritis. The most convincing results seem to come from the differentiation of active (rheumatoid) arthritis from controls or from other types of inflammatory processes. ${ }^{36,37}$

Results from a brain study ${ }^{25}$ have shown that most of the types of shapes occurred in most gliomas, but that the TIC shape analysis, in contrast to PKM analysis, was not able to highlight the effect of antiangiogenic treatment on high grade glioma. This is one of the few examples of work where the TIC shape analysis has been applied in the follow-up of patient treatment. Authors investigating the effect of therapy, ie, Wang et a ${ }^{32}$ (antiangiogenic treatment in liver hepatocellular carcinoma), Lavini et al, ${ }^{25}$ and Ziech et $\mathrm{al}^{41}$ (antitumor necrosis factor-alpha in fistulas) agree that the TIC shape does not highlight the effect of the antiangiongenic drugs.

In one other article using TIC analysis, but not using the TIC shape as a diagnostic tool, a change in TIC shape was mentioned. ${ }^{44}$ The authors, who use the steepest slope to monitor response to preoperative chemotherapy in MSK tumors, conclude that steepest slope is not useful for discrimination between benign and malignant tumors due to excessive overlap, but that the TICs they present for two example patients change from a type Ff into a type FS after chemotherapy in one patient, and show a clear type SS in a patient responding well to chemotherapy.

\section{Discussion}

Literature using TIC shape analysis is growing, but it is still fragmented. Unlike its (twin) application in breast imaging, where authors seem to have agreed on a classification standardization using the three points method, ${ }^{14}$ the TIC shape analysis in other parts of the body seems to present a diversity which makes it extremely challenging to compare the results of the different studies. Nevertheless, a common thread can be found in this diversity: in most publications, malignant processes seem to present, in one way or the other (prevalence or simple occurrence), with a type shape Ff, and most benign lesions present with a prevalence of type SS. Unfortunately, however, the opposite has been observed in certain tumor types where the TIC shape analysis does not yet show enough sensitivity and specificity. Part of this problem has been addressed by adding spatial resolution through the pixel by pixel analysis. In this way, by changing the results derived from a single averaged TIC to mentioning the prevalence or relative ratio of a certain shape and observing the heterogeneity of the distribution, ${ }^{23,45}$ it was seen that malignant lesions (chondrosarcoma), which would have been classified as type SS on an ROI based approach, presented indeed a significant $\mathrm{Ff}$ component. ${ }^{23}$

Most recent studies use the pixel by pixel method, and it is expected that this will soon completely replace the ROI analysis, also as software to perform TIC shape analysis becomes more available. ${ }^{46,47}$ Only in this way will it be possible to approach the sensitivity and specificity of other quantitative techniques, such as PKM.

Certainly another problem has to be addressed, ie, the number of classes used by each study. It is still difficult on the basis of the published studies to assess which classes are truly necessary, or whether there are classes that could be removed. Conversely, it is possible that more classes might have to be added. Works on automatic detection of the classes that "naturally" occur in DCE-MRI datasets are still in progress, for example the work using PCA. ${ }^{48}$

\section{Limitations of the TIC shape analysis and dependence on MRI scan parameters}

The TIC shape analysis does not provide absolute measures. It is dependent, although only slightly, on the protocol chosen: the length of the DCE-MRI scan, the time resolution, and the scan parameters (TR/flip angle) can all influence the final shape. Because the T1 weighting of the sequence determines the relationship between signal and contrast agent concentration, ${ }^{1}$ situations where signal saturation occurs due to high contrast agent concentrations should be avoided. If signal saturation occurs (for example due to insufficient T1 weighting), part of the enhancement curve might reach a plateau (type FP) instead of showing an increasing-decreasing pattern (type Fs). This can have an effect on the shape classification, resulting in the same tissue possibly being classified differently when using different protocols. Reproducibility studies in this field would be very welcome.

Partial volume is also a problem in DCE-MRI protocols that privilege time resolution over spatial resolution (voxels can be of the order of 1.5 to 3 millimeters [in plane] and 3 to 6 millimeters in the "slice" direction). The coarse resolution might result in voxels hosting different TIC shape types. As an example, voxels with mixed type $\mathrm{V}$ (vessel) and type SS might result in an averaged type Ff. This could be the case in, among others, the synovial membrane which is fed by a neighboring artery. In this case, it is still difficult to discriminate between voxels originating purely from intraand extravascular compartments. 
Other problems involve the temporal resolution. If insufficient, the arterial/venous type $\mathrm{V}$ which is characterized by an early steep slope followed by fast washout might not be recognized at all. Typically, when the contrast agent is injected as a short bolus, the initial peak typically lasts less than 40 seconds. ${ }^{49,50}$ It is to be expected, therefore, that a temporal resolution of less than 40 seconds will not be able to identify the initial enhancement in the vessel.

Despite the advantages of the pixel by pixel approach in terms of sensitivity to spatial heterogeneity, the technique suffers more from the lower signal to noise ratio, as well as from patient movement. The dependence of the automatic classification on pixel size has not been investigated yet. Motion correction has only been used in one article ${ }^{40}$ where the TIC shape analysis (pixel by pixel) was applied in the bowel. The usefulness of motion correction in improving the TIC shape analysis has not been addressed in any of the studies presented here. Furthermore, the dependence of the classification on the particular Gd-based contrast agent has also not been investigated.

\section{Comparison with pharmacokinetic (PK) analysis}

When compared to the quantitative PK analysis, the advantage of the TIC shape analysis lies predominantly in its simplicity and accessibility. Although PKM has been acknowledged to provide the best MR endpoint for assessing solid tumors, neoangiogenesis, and response to antiangiogenic therapy, ${ }^{4}$ its difficult implementation, involving the extra measurement of the native T1, the calculation of the absolute contrast agent concentrations, as well as an AIF has put off many clinically oriented researchers who still prefer the more heuristic, but more accessible, TIC shape analysis approach. Furthermore, although a correctly implemented PKM analysis can add significant information to the tissue physiology, the chance to implement it incorrectly is not negligible. It is widely acknowledged that a small error in the AIF can propagate in the resulting $\mathrm{K}^{\text {trans }}$ and $\mathrm{v}_{\mathrm{e}}$ parameters of the PKM in a significant way. In the same way any inaccuracy in the value of the relaxivity and of the T1 will also severely affect the final $\mathrm{K}^{\text {trans }}{ }^{51}$ The robustness and reproducibility of the method, therefore, suffers. The TIC shape analysis, on the contrary, is not dependent on any assumption; it only relies on the observation and classification of the raw data.

\section{Future directions}

The quest to assess the clinical value of the TIC shape is still open. Although the number of applications has grown, there is a need for more focused studies and for a more general consensus on the protocols to be used.

It has been shown that the TIC shape analysis alone might not be able to highlight some effects, such as the antiangiogenic effects of drugs, ${ }^{25}$ and the method might need to be refined to improve its sensitivity.

The necessity of devising a new quantitative non-PKM based analysis method has been widely recognized and new model-free methods continue to appear. Among those, there are methods that continue to rely on the classifications of TIC shapes or on parameters which are derived from them. ${ }^{43,48}$ Guo and Reddick ${ }^{52}$ describe a way of redefining shapes and extracting descriptive parameters from them. They proposed a method called curve pattern analysis (CPA) which is used to generate some "quantitative" CPA parameters, eg, K, beta1, beta2, and beta3, which quantify in some way the TIC shape. These parameters were tested in pediatric osteosarcomas.

Moreover, pure TIC shape analysis has been approached with different image analysis techniques, more independent of operator chosen thresholds. In the last year, a large amount of literature has appeared that makes use of PR techniques to classify DCE-MRI data. PR techniques involve automatic recognition of certain enhancement patterns based on a statistical classifier, which is responsible for decision making to assign a certain pattern to a certain class. Many types of classifiers exist and the literature describes the use of various classifiers for the analysis of DCE-MRI data. Artificial neural networks are used by Kale et al, ${ }^{53}$ Lucht et al, ${ }^{54}$ and Nattkemper et al, ${ }^{55}$ and support vector machines by Levman et al. ${ }^{56}$ A good overview of the methods applied to DCE-MRI can be found in Eyal and Degani. ${ }^{43}$ Most of these works have been carried out in breast lesions.

It is beyond the scope of this review to dwell on the PR techniques and their methodology. Still, it is worth mentioning that significant advances have been made in this field toward an improvement of the classification of DCE-MRI data. Importantly, DCE-MRI generated TIC shapes can be combined with other features, arising for example from anatomical structure, to produce an automatic classification of tissues into malignant and benign. Especially in the field of breast imaging, the works using combined features have reached an advanced stage. New and interesting methods have been proposed, such as textural kinetic analysis. ${ }^{57}$ It is desirable that the new developments in breast DCE-MRI analysis will soon be adapted to the data acquired with higher temporal resolution described in this review, and possibly applied in other diseases. 


\section{Conclusion}

Because not only the protocols differ greatly in terms of sequence and sequence parameters, and because the classification systems appear so diverse, standardization should eventually be proposed, in a way similar to that established for breast imaging. The effect of standardization will result in easier comparison between studies, and make meta-analysis possible. The key issue as to whether the TIC shape analysis alone can differentiate malignant from benign tumors, or differentiate between tumor grades, remains controversial. Besides the heterogeneous clinical results, the plethora of different names for the different shapes and lack of standardization contribute to the uncertainty. Nevertheless, from this overview we could find agreement that a prevalence of a rapidly growing, rapidly washing out TIC shape remains consistently a mirror of malignancy in tumors and of activity in rheumatoid arthritis, the latter being a pathology where TIC shape analysis seems to be particularly successful. The few studies investigating the effect of drugs did not show the technique to be promising. It is to be hoped that an increase in the use of spatially resolved TIC shape analysis techniques will lead to more insight in the tissue behavior, and that a combination with other analysis techniques might increase its sensitivity.

\section{Disclosure}

The authors report no conflict of interest in this work.

\section{References}

1. Evelhoch JL. Key factors in the acquisition of contrast kinetic data for oncology. J Magn Reson Imaging. 1999;10(3):254-259.

2. Tofts PS, Brix G, Buckley DL, et al. Estimating kinetic parameters from dynamic contrast-enhanced T(1)-weighted MRI of a diffusable tracer: standardized quantities and symbols. J Magn Reson Imaging. 1999;10(3):223-232.

3. Padhani AR, Leach MO. Antivascular cancer treatments: functional assessments by dynamic contrast-enhanced magnetic resonance imaging. Abdom Imaging. 2005;30(3):324-341.

4. Leach MO, Brindle KM, Evelhoch JL, et al; Pharmacodynamic/Pharmacokinetic Technologies Advisory Committee, Drug Development Office, Cancer Research UK. The assessment of antiangiogenic and antivascular therapies in early-stage clinical trials using magnetic resonance imaging: issues and recommendations. Br J Cancer. 2005;92(9):1599-1610.

5. Henderson E, Rutt BK, Lee TY. Temporal sampling requirements for the tracer kinetics modeling of breast disease. Magn Reson Imaging. 1998;16(9):1057-1073.

6. Kaiser WA, Zeitler E. MR imaging of the breast: fast imaging sequences with and without Gd-DTPA. Preliminary observations. Radiology. 1989; 170:681-686.

7. Hauth EA, Stockamp C, Maderwald S, et al. Evaluation of the threetime-point method for diagnosis of breast lesions in contrast-enhanced MR mammography. Clin Imaging. 2006;30(3):160-165.

8. Kubassova O, Boesen M, Boyle RD, et al. Fast and robust analysis of dynamic contrast enhanced MRI datasets. Med Image Comput Comput Assist Interv. 2007;10(2):261-269.
9. van Rijswijk CS, Hogendoorn PC, Taminiau AH, Bloem JL. Synovial sarcoma: dynamic contrast-enhanced MR imaging features. Skeletal Radiol. 2001;30(1):25-30.

10. Van der Woude HJ, Verstraete KL, Hogendoorn PC, Taminiau AH, Hermans J, Bloem JL. Musculoskeletal tumors: does fast dynamic contrast-enhanced subtraction MR imaging contribute to the characterization? Radiology. 1998;208(3):821-828.

11. Verstraete KL, Lang P. Bone and soft tissue tumors: the role of contrast agents for MR imaging. Eur J Radiol. 2000;34(3):229-246.

12. Lavini C, de Jonge MC, van de Sande MG, Tak PP, Nederveen AJ, Maas M. Pixel-by-pixel analysis of DCE MRI curve patterns and an illustration of its application to the imaging of the musculoskeletal system. Magn Reson Imaging. 2007;25:604-612.

13. Yuan J, Chow SK, Yeung DK, King AD. A five-colour colour-coded mapping method for DCE-MRI analysis of head and neck tumors. Clin Radiol. 2012;67(3):216-223.

14. Degani H, Gusis V, Weinstein D, Fields S, Strano S. Mapping pathophysiological features of breast tumors by MRI at high spatial resolution. Nat Med. 1997;3(7):780-782.

15. Massurakis S, Gibbs P, Horsman A. Primary breast abnormalities: selective pixel sampling on dynamic gadolinium-enhanced MR images. Radiology. 1998;206:465-473.

16. Rieber A, Brambs HJ, Gabelmann A, Helmann V, Kreienberg R, Kuhn T. Breast MRI for monitoring response of primary breast cancer to neoadjuvant chemotherapy. Eur Radiol. 2002;12:1711-1719.

17. Preim U, Glaßer S, Preim B, Fischbach F, Ricke J. Computer-aided diagnosis in breast DCE-MRI - quantification of the heterogeneity of breast lesions. Eur J Radiol. 2012;81(7):1532-1538.

18. Turnbull LW. Dynamic contrast-enhanced MRI in the diagnosis and management of breast cancer. NMR Biomed. 2009;22(1):28-39.

19. Hawighorst H, Libicher M, Knopp MV, Moehler T, Kauffmann GW, Kaick GV. Evaluation of angiogenesis and perfusion of bone marrow lesions: role of semiquantitative and quantitative dynamic MRI. J Magn Reson Imaging. 1999;10(3):286-294.

20. van Rijswijk CS, Geirnaerdt MJ, Hogendoorn PC, et al. Soft-tissue tumors: value of static and dynamic gadopentetate dimeglumineenhanced MR imaging in prediction of malignancy. Radiology. 2004;233:493-502.

21. Tokuda O, Hayashi N, Taguchi K, Matsunaga N. Dynamic contrastenhanced perfusion MR imaging of diseased vertebrae: analysis of three parameters and the distribution of the time-intensity curve patterns. Skeletal Radiol. 2005;34(10):632-638.

22. Kawakami Y, Kunisada T, Sugihara S, et al. New approach for assessing vascular distribution within bone tumors using dynamic contrastenhanced MRI. J Cancer Res Clin Oncol. 2007;133(10):697-703.

23. Lavini C, Pickaart BC, de Jonge MC, Schaap GR, Maas M. Region of interest and pixel-by-pixel analysis dynamic contrast enhanced magnetic resonance imaging parameters and time-intensity curve shapes: a comparison in chondroid tumors. Magn Reson Imaging. 2009;27:62-68.

24. Sasaki M, Sumi M, Eida S, et al. Multiparametric MR imaging of sinonasal diseases: time-signal intensity curve- and apparent diffusion coefficient-based differentiation between benign and malignant lesions. Am J Neuroradiol. 2011;32(11):2154-2159.

25. Lavini C, Verhoeff JJ, Majoie CB, Stalpers LJ, Richel DJ, Maas M. Model-based, semiquantitative and time intensity curve shape analysis of dynamic contrast-enhanced MRI: a comparison in patients undergoing antiangiogenic treatment for recurrent glioma. J Magn Reson Imaging. 2011;34(6):1303-1312.

26. Eida S, Ohki M, Sumi M, Yamada T, Nakamura T. MR factor analysis: improved technology for the assessment of 2D dynamic structures of benign and malignant salivary gland tumors. J Magn Reson Imaging. 2008;27(6):1256-1262.

27. Yabuuchi H, Matsuo Y, Kamitani T, et al. Parotid gland tumor: can addition of diffusion-weighted MR-imaging to dynamic contrast-enhanced MR imaging improve diagnostic accuracy in characterization? Radiology. 2008;249(3):909-916. 
28. Schaefer JF, Schneider V, Vollmar J, et al. Solitary pulmonary nodules: association between signal characteristics in dynamic contrast enhanced MRI and tumor angiogenesis. Lung Cancer. 2006;53(1):39-49.

29. Donmez FY, Yekeler E, Saeidi V, Tunaci A, Tunaci M, Acunas G. Dynamic contrast enhancement patterns of solitary pulmonary nodules on 3D gradient-recalled echo MRI. AJR Am J Roentgenol. 2007;189(6):1380-1386.

30. Kono R, Fujimoto K, Terasaki H, et al. Dynamic MRI of solitary pulmonary nodules: comparison of enhancement patterns of malignant and benign small peripheral lung lesions. AJR Am J Roentgenol. 2007;188(1):26-36.

31. Yamashita Y, Fan ZM, Yamamoto H, et al. Spin-echo and dynamic gadolinium-enhanced FLASH MR imaging of hepatocellular carcinoma: correlation with histopathologic findings. J Magn Reson Imaging. 1994;4(1):83-90.

32. Wang J, Chen LT, Tsang YM, Liu TW, Shih TT. Dynamic contrastenhanced MRI analysis of perfusion changes in advanced hepatocellular carcinoma treated with an antiangiogenic agent: a preliminary study. AJR Am J Roentgenol. 2004;183(3):713-719.

33. Tuncbilek N, Karakas HM, Altaner S. Dynamic MRI in indirect estimation of microvessel density, histologic grade, and prognosis in colorectal adenocarcinomas. Abdom Imaging. 2004;29(2):166-172.

34. Noworolski SM, Henry RG, Vigneron DB, Kurhanewicz J. Dynamic contrast-enhanced MRI in normal and abnormal prostate tissues as defined by biopsy, MRI, and 3D MRSI. Magn Reson Med. 2005;53(2): 249-255.

35. Kubassova O, Boyle R, Radjenovic A. Quantitative analysis of dynamic contrast enhanced MRI datasets of the metacarpophalangeal joints. Acad Radiol. 2007;14:1189-1200.

36. van der Leij C, van de Sande MG, Lavini C, Tak PP, Maas M. Rheumatoid synovial inflammation: pixel-by-pixel dynamic contrastenhanced MR imaging time-intensity curve shape analysis - a feasibility study. Radiology. 2009;253(1):234-240.

37. van de Sande MG, van der Leij C, Lavini C, Wijbrandts CA, Maas M, Tak PP. Characteristics of synovial inflammation in early arthritis analysed by pixel-by-pixel time-intensity curve shape analysis. Rheumatology. 2012;51(7):1240-1245.

38. Kim EY, Kwack KS, Cho JH, Lee DH, Yoon SH. Usefulness of dynamic contrast-enhanced MRI in differentiating between septic arthritis and transient synovitis in the hip joint. AJR Am J Roentgenol. 2012;198(2): $428-433$.

39. Giusti S, Faggioni L, Neri E, et al. Dynamic MRI of the small bowel: usefulness of quantitative contrast-enhancement parameters and timesignal intensity curves for differentiating between active and inactive Crohn's disease. Abdom Imaging. 2010;35(6):646-653.

40. Ziech ML, Lavini C, Caan MW, et al. Dynamic contrast-enhanced MRI in patients with luminal Crohn's disease. Eur J Radiol. 2012;81(11): 3019-3027.

41. Ziech ML, Lavini C, Bipat S, et al. Dynamic contrast-enhanced MRI in determining disease activity in perianal fistulizing Crohn's disease: a pilot study. AJR Am J Roentgenol. 2013;200(2):170-177.

42. Horsthuis K, Lavini C, Bipat S, Stokkers PC, Stoker J. Perianal Crohn's disease: evaluation of dynamic contrast-enhanced MR imaging as an indicator of disease activity. Radiology. 2009;251(2):380-387.

43. Eyal E, Degani H. Model based and model free parametric analysis of breast dynamic contrast enhanced MRI. NMR Biomed. 2009;22:40-53.

Reports in Medical Imaging

\section{Publish your work in this journal}

Reports in Medical Imaging is an international, peer-reviewed, open access journal publishing original research, reports, reviews and commentaries on all areas of medical imaging. The manuscript management system is completely online and includes a very quick and fair peer-review system, which is all easy to use.
44. Kajihara M, Sugawara Y, Sakayama K, Kikuchi K, Mochizuki T, Murase $\mathrm{K}$. Evaluation of tumor blood flow in musculoskeletal lesions: dynamic contrast-enhanced MR imaging and its possibility when monitoring the response to preoperative chemotherapy-work in progress. Radiat Med. 2007;25(3):94-105.

45. El Khouli RH, Macura KJ, Kamel IR, Jacobs MA, Bluemke DA. 3-T dynamic contrast-enhanced MRI of the breast: pharmacokinetic parameters versus conventional kinetic curve analysis. AJR Am J Roentgenol. 2011;197(6):1498-1505.

46. Lavini C, Maas M. DCE-MRI analysis package comprising pixel-bypixel classification of Time Intensity Curves shapes, permeability maps and Gd concentration calculation. Abstract from the ESMRMB 2008 Congress, October 2-4, 2008; Valencia, Spain. Magn Reson Materials Physics Biol Med. 2008:21Suppl1, S1:486.

47. Kubassova O, Boesenb M, Cimmino M, Bliddal H. A computer-aided detection system for rheumatoid arthritis MRI data interpretation and quantification of synovial activity. Eur J Radiol. 2010;74(3):e67-e72.

48. Eyal E, Bloch BN, Rofsky NM, et al. Principal component analysis of dynamic contrast enhanced MRI in human prostate cancer. Invest Radiol. 2010;45:174-181.

49. Andersen C, Taagehøj JF, Mühler A, Rehling M. Approximation of arterial input curve data in MRI estimation of cerebral blood-tumorbarrier leakage: comparison between Gd-DTPA and 99 mTc-DTPA input curves. Magn Reson Imaging. 1996;14(3):235-241.

50. Parker GJ, Roberts C, Macdonald A, et al. Experimentally-derived functional form for a population- averaged high-temporal-resolution arterial input function for dynamic contrast-enhanced MRI. Magn Reson Med. 2006;56(5):993-1000.

51. Dale BM, Jesberger JA, Lewin JS, Hillenbrand CM, Duerk JL. Determining and optimizing the precision of quantitative measurements of perfusion from dynamic contrast enhanced MRI. J Magn Reson Imaging. 2003;18(5):575-584.

52. Guo J-Y, Reddick WE. DCE-MRI Pixel by Pixel quantitative Curve Pattern Analysis and its application to osteosarcoma. J Magn Res Imaging. 2009;30:177-184.

53. Kale MC, Clymer BD, Koch RM, et al. Multispectral co-occurrence with three random variables in dynamic contrast enhanced magnetic resonance imaging of breast cancer. IEEE Trans Med Imaging. 2008;27(10):1425-1431.

54. Lucht RE, Delorme S, Hei J, et al. Classification of signal-time curves obtained by dynamic magnetic resonance mammography: statistical comparison of quantitative methods. Invest Radiol. 2005;40(7):442-447.

55. Nattkemper TW, Arnrich B, Lichte O, et al; UK MARIBS Breast Screening Study. Evaluation of radiological features for breast tumour classification in clinical screening with machine learning methods. Artif Intell Med. 2005;34(2):129-139.

56. Levman J, Leung T, Causer P, Plewes D, Martel AL. Classification of dynamic contrast-enhanced magnetic resonance breast lesions by support vector machines. IEEE Trans Med Imaging. 2008;27(5):688-696.

57. Agner SC, Soman S, Libfeld E, et al. Textural kinetics: a novel dynamic contrast-enhanced (DCE)-MRI feature for breast lesion classification. $J$ Digit Imaging. 2011;24(3):446-463.

Visit http://www.dovepress.com/testimonials.php to read real quotes from published authors. 